\title{
La production de céruse en France au XIXe siècle : évolution d'une industrie dangereuse
}

Laurence Lestel

\section{(2) OpenEdition}

12 Journals

Édition électronique

URL : https://journals.openedition.org/tc/1067

DOI : $10.4000 /$ tc. 1067

ISSN : 1952-420X

Éditeur

Éditions de l'EHESS

\section{Édition imprimée}

Date de publication : 1 mars 2002

ISSN : 0248-6016

\section{Référence électronique}

Laurence Lestel, «La production de céruse en France au XIXe siècle : évolution d'une industrie dangereuse », Techniques \& Culture [En ligne], 38 | 2002, mis en ligne le 10 février 2006, consulté le 29 septembre 2022. URL : http://journals.openedition.org/tc/1067 ; DOI : https://doi.org/10.4000/tc.1067

Ce document a été généré automatiquement le 29 septembre 2022.

Tous droits réservés 


\title{
La production de céruse en France au XIXe siècle : évolution d'une industrie dangereuse
}

\author{
Laurence Lestel
}

1 Depuis la fin du XVIIIe siècle, la demande en céruse pour blanchir les murs s'accroît. Or, si quelques entrepreneurs s'essaient à la fabrication de ce produit dès la fin des années 1780, la céruse est essentiellement importée de Hollande et d'Angleterre. Il n'y aura de production significative en France qu'à partir de l'installation de l'usine de Clichy en 1809, puis de plusieurs sites à Lille dans les années 1820. Cette industrie est reconnue comme dangereuse avant même son développement en France, comme l'atteste ce commentaire du Conseil de Salubrité de la Seine en 1824 :

«De tout temps la fabrication de la céruse a donné lieu à des accidents extrêmement graves [...]. En Hollande, où la céruse se fabrique en très grande quantité, le tiers des ouvriers employés à cette opération périt chaque année. ${ }^{1}$

Dès 1848, une première campagne étatique tente de substituer, au blanc de plomb, le blanc de zinc - un oxyde de zinc non toxique- sans que l'on observe le moindre fléchissement dans la courbe de production de la céruse, soutenue par une forte demande depuis que le blanchiment des murs a cours jusque dans les moindres villages $^{2}$. La production et la consommation françaises ne cessent de croître jusqu'à la Première Guerre mondiale, alors que la première loi qui restreint les usages de la céruse sera votée en 1909.

3 L'objet de cette étude est de montrer comment les producteurs de céruse, conscients de la dangerosité de cette activité, ont réagi aux sollicitations extérieures, notamment étatiques, en modifiant au cours du XIXe siècle les différentes étapes de la fabrication par des innovations sous-tendues par la volonté de rendre le procédé moins dangereux pour les ouvriers. L'ennemi était la poussière de céruse. Nous présenterons tour à tour les producteurs de céruse, le procédé de fabrication hollandais et son impact sur la santé de l'ouvrier, les améliorations apportées par les producteurs, le cas particulier de l'usine de Clichy et enfin la manière dont le problème a été géré par l'État. 
Les producteurs de céruse

Comme beaucoup des métiers ayant trait au plomb, la fabrication de la céruse est soumise à déclaration dès le premier décret concernant les établissements dangereux, insalubres ou incommodes du 15 octobre 1810. La céruse, ou blanc de plomb, est intégrée à la classe 2 à cause de ses émanations dangereuses. À cette date, n'existe qu'un seul établissement d'envergure, celui de Clichy, fondé en 1809, et dont la production, prévue pour être de l'ordre de 600 tonnes, doit couvrir un tiers des besoins français. On trouve cependant la trace de nombreux petits établissements qui ont tenté de développer le procédé importé de Hollande, par, dit-on, M. Chaillot de Paris, en $1791^{3}$. Ces établissements se montent à Paris, Orléans, Giver, Rouen et Pezenas. Très vite, une région prend le dessus, tant par le nombre de fabriques qui s'y crée que par la qualité de la céruse produite: celle de Lille et de quelques communes voisines (Wazemmens, Esquermes, Fives). La figure 1 indique le nombre de fabriques de céruse dans les départements de la Seine et du Nord, tel qu'il a été calculé annuellement à partir des dates d'apparition et de disparition des entreprises lilloises et parisiennes, ainsi que le nombre total des entreprises françaises après diverses sources ponctuelles ${ }^{4}$.

5 Alors que le nombre d'usines reste stable -voire diminue- dans le reste de la France, celui de la région lilloise augmente entre les années 1820 et 1840, puis entre 1865 et 1885 , jusqu'à occuper une place prédominante dans le paysage français. En 1908, les statistiques des Etablissements insalubres répertorient 19 fabricants de céruse en France, dont 10 à Lille, 3 en Haute-Garonne et un seul dans les départements de la Seine, Seine-et-Oise, Somme, Loire-Inférieure, Meuse et Rhône ${ }^{5}$. Quelle que soit la qualité de ces statistiques départementales (deux fabriques de céruse de Marseille sont ainsi omises car elles ont probablement été répertoriées comme fonderies de plomb), la supériorité numérique de Lille est flagrante. Elle est d'ailleurs reconnue par la progression: "Lille est le centre français de la production de la céruse, car, à part quelques usines semées dans tout le reste de la France, la capitale de la Flandre semble s'être réservée le monopole de cette fabrication ", nous rapporte un héritier de l'usine de la Veuve Perus en 1900 (Dubreucq 1900). Ou bien, dit encore plus péremptoirement l'« Inspecteur de l'insalubrité » lillois Thibaut : «Il n'y a qu'à Lille où l'on rencontre ce genre d'industrie $"^{6}$. Toutes les entreprises lilloises n'ont pas la même importance. Sur les 20 répertoriées dans l'Almanach du Commerce, 14 ont des durées d'activité avérées supérieures à dix ans, la plupart ayant des existences très stables. La première est celle de Louis Faure, fondée en 1819, mais le leader est incontestablement Théodore Lefebvre qui produit entre 35 et $40 \%$ de la céruse lilloise de 1830 à 1850 (Lefebvre et Cie 1865 : $143)^{7}$. C'est l'usine de la Vve Perus à Fives, dont la production démarre en 1858, qui est citée en exemple par Turgan en 1885, elle est le plus gros producteur de Lille (3500 tonnes annuelles contre 3000 pour Th. Lefebvre). L'usine modèle du début du XXe siècle est celle d'Expert-Bezançon qui, après avoir racheté Faure, transfère ses activités à Saint-André-lez-Lille en 1900 (Bouchonnet 1909).

6 Lille domine le secteur de la céruse non seulement par le nombre de la fabriques qui s'y trouve, mais aussi par la quantité de céruse qui y est produite: en 1852, plusieurs sources citent le chiffre de $5 / 6^{\text {e }}$ de la production française ${ }^{8}$. Un tableau de la production réalisé pour l'année 1850 donne un chiffre légèrement inférieur, puisque 7 fabriques à Lille produisent ensemble 4600 tonnes de céruse (dont 1800 par Th. Lefebvre luimême), contre 1600 tonnes produites par les deux usines de Paris et celle de Tours; les autres Fabriques, de Marseille et de Strasbourg, considérées comme insignifiantes, 
fournissent à peine 300 tonnes (Lefebvre et cie 1865 : 143-148). La figure 2 reporte la quantité de céruse produite par la région lilloise et en France ainsi que les importations et exportations de céruse sur la période de 1800-1930. Les importations de céruse de Hollande et d'Angleterre cessent dès que la production française devient significative : celles de Hollande chutent de plus de 1000 tonnes annuelles dans les années 1820 à moins de 100 tonnes en 1832 (Kuhlmann 1834: 30; Lefebvre et Cie 1865:3). L'importance de la production lilloise est explicite jusqu'en 1880. Nous ne disposons pas de chiffres distinguant la production de Lille de celle de la France après cette date, probablement parce que les deux chiffres sont en fait confondus : l'usine de Clichy ne produit plus de céruse après 1882 et Bezançon, qui produisait 1300 tonnes de céruse en 1866 (Bezançon frères 1867 : 5), soit trois fois moins que les gros producteurs lillois, transfère la majeure partie de ses activités à Lille lors du achat de Faure. Les autres fabriques françaises sont toujours considérées comme négligeables.

7 À la fin du XIXe siècle, environ un tiers du plomb neuf extrait ou importé en France chaque année sert à produire de la céruse (Faure 1889); pourtant, le nombre d'ouvriers employés dans ce secteur d'activité reste faible : estimé à 450 pour toute la France en 1836, il s'élève à une centaine d'ouvriers chez Théodore Lefebvre au milieu du siècle ou chez la Vve Perus vers 1885. En 1876, on compte 388 ouvriers lillois, d'après l'inspecteur du Conseil de salubrité Meurein, et 304 en 1878, d'après Gautier. Le Dr Arnould dénombre près d'un millier d'ouvriers cérusiers employés dans 10 céruseries lilloises en 1878, mais ce chiffre semble excessif dans la mesure où à notre connaissance, aucune des céruseries n'emploie plus de 75 ouvriers à cette date ${ }^{9}$.

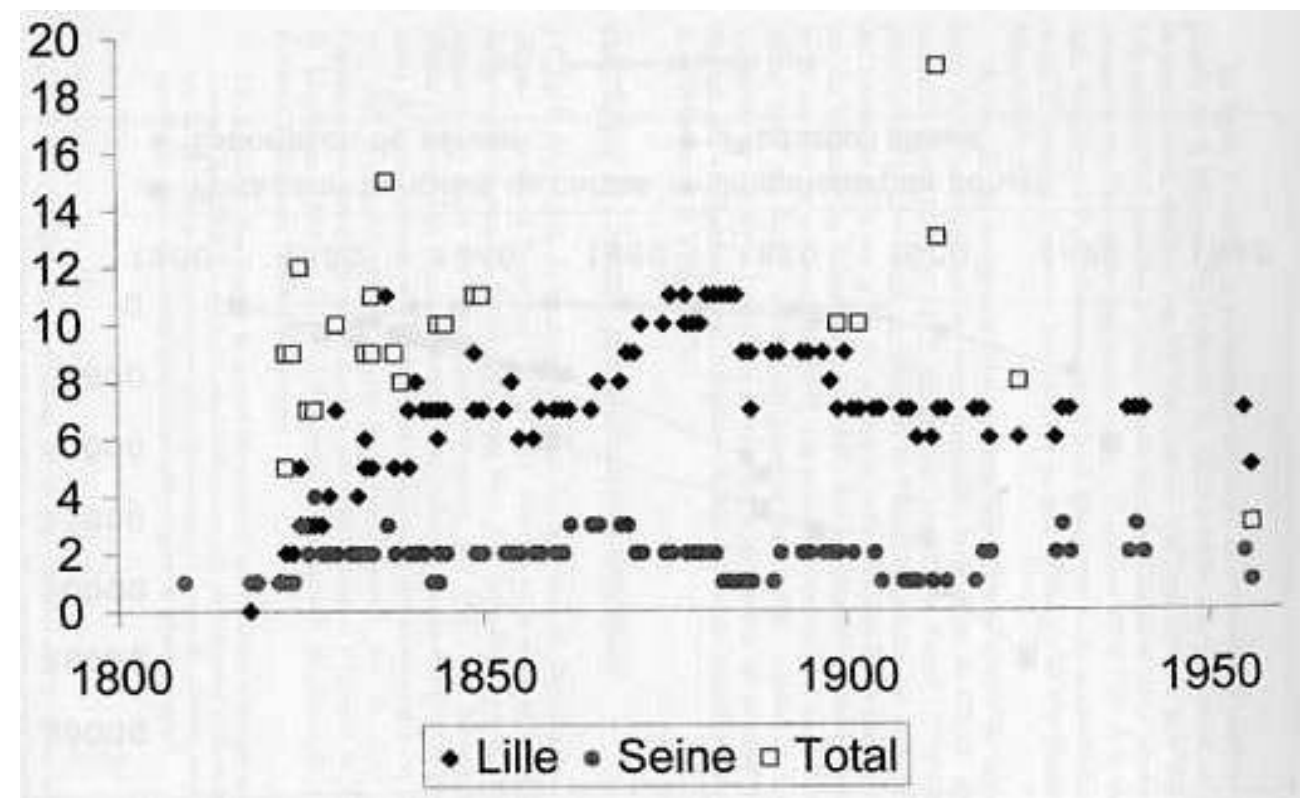


Figure 1. Nombre de fabriques de céruse en France

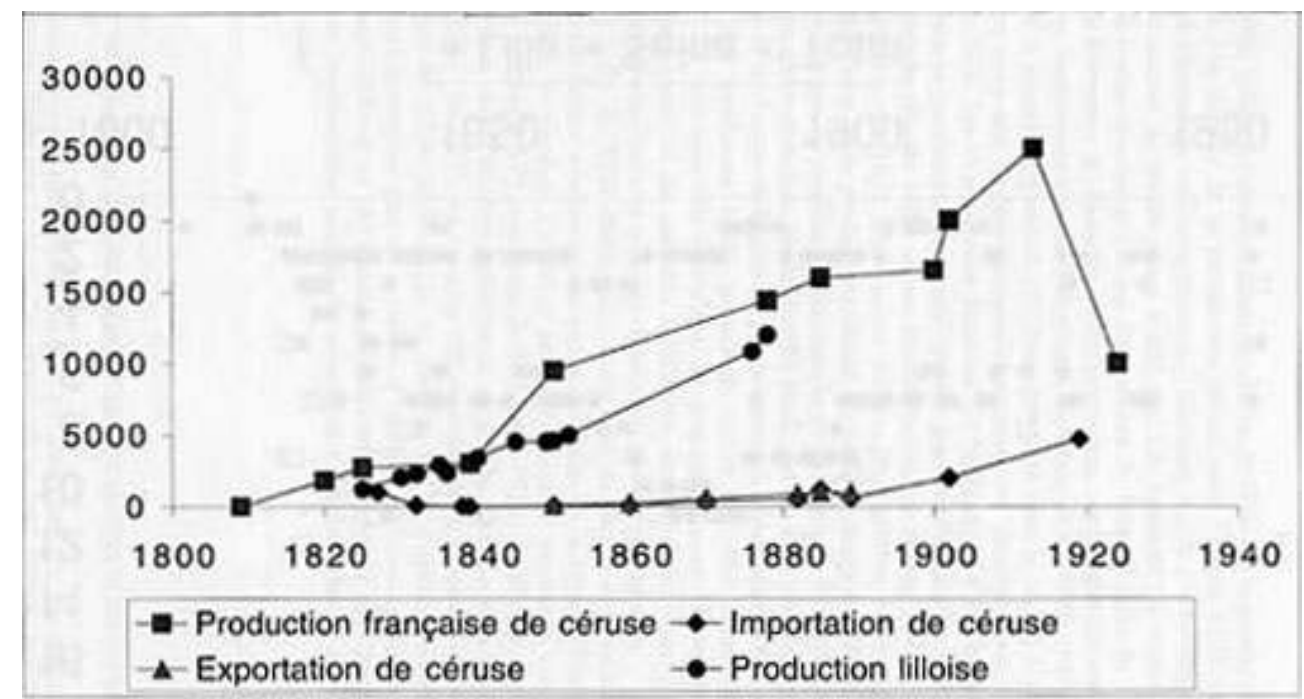

Figure 2. Production française de céruse

8 La figure 3 montre la production de céruse et le nombre d'ouvriers employés par la fabrique Th. Lefebvre de 1825 à 1890 . On y constate un gain en productivité, souligné par Th. Lefebvre en 1867, qui estimait qu'il était dû à la stabilité des ouvriers « qu'un salaire relativement élevé nous permet de conserver régulièrement dans notre usine " (Lefebvre 1878 :9). À la même date, Bezançon, à Paris, constatait qu'il avait quasiment triplé sa production et divisé le nombre d'ouvriers par deux en un peu moins de 20 ans (Bezançon frères 1867 : 5).

9 À côté de ces entreprises lilloises, deux fabricants de céruse du département de la Seine ont retenu notre attention: la fabrique de Clichy, opérationnelle entre 1809 et 1882, dirigée par Jean-Louis Roard (1774-1873), puis par son gendre Orsat, ancien élève de l'École Polytechnique et de l'École des Mines, et enfin par le fils de ce dernier; celle d'Expert-Bezançon, installée rue du Château des rentiers depuis 1844. Elles emploient respectivement 60 et 30 ouvriers en moyenne, en 1870. De toutes ces entreprises, seule la pionnière, celle de Roard, utilise un procédé particulier, justement appelé « procédé de Clichy». Les autres fabriquent la céruse par un procédé traditionnel, dit "hollandais ", qui n'a guère changé depuis ses premières descriptions (Homburg \& Vlieger 1996).

Fabriquer la Céruse : une industrie dangereuse

"Dans la fabrication de la céruse, on peut dire que tout est dangereux ", nous affirme Charles de Freycinet (1870:72). Mais la prise de conscience de la dangerosité du procédé de fabrication est bien plus précoce si l'on en croit les rapports des jurys des expositions françaises. En 1827, des fabricants de céruse s'étaient déjà attachés à rendre leur industrie moins dangereuse pour la santé de leurs ouvriers et «les louables sollicitudes des fabricants à cet égard ont été particulièrement prises en considération par le jury dans l'appréciation des droits qu'ils avaient aux récompenses » (Héricart de Thury \& Migneron $1827: 124)$. À l'Exposition industrielle de Paris en 1834, Frédéric Kuhlmann (1834: 30) précise :

«Il est à désirer dans l'intérêt de la santé (des ouvriers), que l'exemple donné par quelques fabricants d'attacher des médecins aux établissements soit généralement 
suivi, et surtout que les fabricants de céruse mettent moins d'insouciance à écarter des ouvriers, autant que possible, les causes des maladies cruelles auxquelles ce genre de travail expose. " ${ }^{10}$

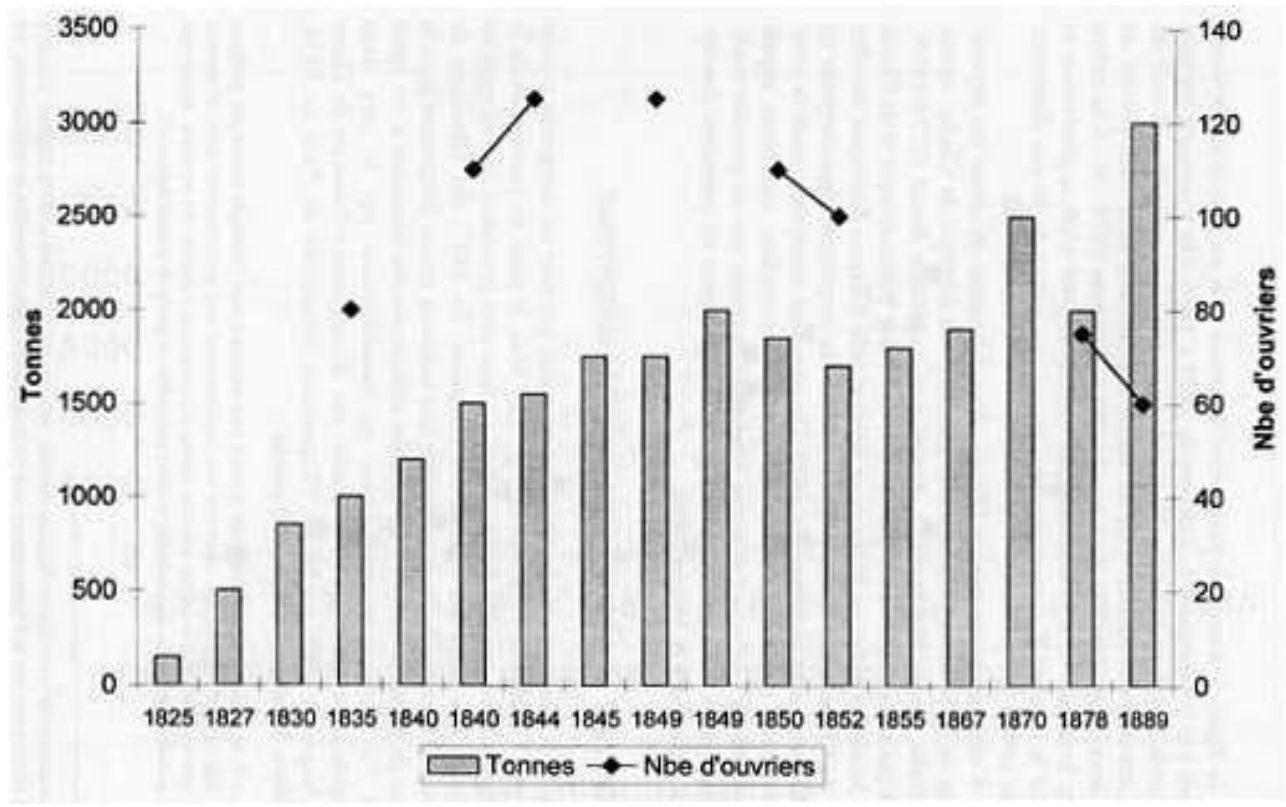

Figure 3. Production de céruse chez Th. Lefebvre entre 1825 et 1889

11 En 1837, Théodore Lefebvre est récompensé à Lille "pour les perfectionnements apportés dans la fabrication de la céruse, afin d'en atténuer l'insalubrité » (Lefebvre et Cie 1865 : 43). En 1844, il est souligné que "dès l'origine de leur fabrique, MM. Th. Lefebvre et Cie se sont attachés à rendre leur industrie moins dangereuse pour la santé de leurs ouvriers $»^{11}$. En 1849, Kuhlmann constate que les Frères Poelman à MoulinsLille « ont fait d'honorables efforts pour rendre moins insalubre le travail de leurs ateliers ", puis, seulement après, indique que "leurs produits sont estimés » (Kuhlmann 1849: 23). "Ce que nous désirons surtout faire apprécier, ce sont les progrès considérables que nous avons fait faire à notre industrie au point de vue de la santé des ouvriers qui travaillent ou emploient la Céruse. Ce but a été celui de toute notre carrière industrielle ", déclare Bezançon pour l'Exposition de 1867 (Bezançon Frères 1867 : 4). À sa mort, le 9 décembre 1872, Théodore Lefebvre a droit aux honneurs de l'Académie Nationale qui souligne combien il «s'est donné pour problème de combattre, toute sa vie, une industrie meurtrière : la rendre salubre et préserver ainsi l'existence d'innombrables ouvriers $»^{12}$. En 1878, on relève à l'Exposition universelle à Paris la présence de la maison L. Faure de Lille, «bien connue pour ses travaux et ses installations spéciales dans le but d'éviter l'intoxication des ouvriers pendant le broyage de la céruse et de recueillir les poussières toxiques entraînées par les ventilateurs » (Engel 1879: 65). Ce type de discours, peut-être convenu, n'est employé qu'au sujet de la céruse. Aucun des autres produits chimiques exposés n'est décrit dans ces termes. Sont décrits leurs qualités, leurs procédés de fabrication, la gestion satisfaisante des résidus, mais seule la céruse est jugée en rapport avec sa toxicité.

Il est vrai que la profession pouvait se faire du souci. Non seulement le nombre de malades de saturnisme provenant des usines de céruse est considérable, mais la proportion de cérusiers admis à l'hôpital par rapport aux ouvriers d'autres branches professionnelles manipulant du plomb ou un de ses dérivés est très élevée. Le nombre 
d'hospitalisations d'ouvriers cérusiers augmente jusqu'à représenter plus de 350 cas en 1846. Puis ce chiffre se stabilise entre 100 et 200 malades par an, avec même une légère dégradation vers la fin de la décennie 1870. La proportion de malades provenant de fabriques de céruse est proche de $65 \%$ sur la période 1838-1815; les autres professionnels hospitalisés sont les peintres qui emploient la céruse comme base de leur peinture. Ce pourcentage passe à $35 \%$ en moyenne pendant la période 1867-1880, pour les ouvriers travaillant soit dans la fabriques de céruse, soit dans les fabriques de minium, et l'on constate que la proportion d'ouvriers peintres augmente sensiblement ${ }^{13}$. Ces chiffres sont à comparer au nombre d'ouvriers employés dans chacun de ces secteurs professionnels. Or, durant toute cette période, n'existent que deux fabriques de céruse d'importance à Paris : celles de Bezançon compte de 30 à 50 ouvriers, et celle de Roard de 45 à 65 . Même si d'autres fabriques ont existé, comme Gruitgens à Saint-Denis dans les années 1860 ou Ozouf à Paris vers 1870, elles semblent n'avoir eu qu'une existence éphémère. Les fabriques de minium emploient 30 ouvriers à Paris vers 1880, d'après Gautier (1883). Nous n'en avons pas retrouvé la trace, à part Ozouf à Paris qui en produit 2,4 tonnes par 24 heures en 1870 (Freycinet $1870: 77,85$ ). Il est cependant à noter que la fabrique de Roard à Clichy est également spécialisée dans la production de ce type de composés du plomb.

13 Ces chiffres conduisent Gautier à réaliser une enquête auprès des bureaux de la Préfecture de Police chargés des intérêts de l'hygiène publique et des chambres syndicales patronales et ouvrières, afin de classer les professions par insalubrité décroissante, en comptant le nombre de saturnins qu'elles fournissent pour 100 ouvriers. Les fabriques de céruse et de minium arrivent largement en tête, avec un pourcentage nettement supérieur à $100^{14}$. Ce pourcentage tombe à $1,8 \%$ pour les peintres en bâtiments qui sont 14000 , ce qui représente 250 malades (Gauier 1883 : 245). Toutes les fabriques de céruse et de minium ne sont d'ailleurs pas dangereuses au même degré. Gautier, comme tous les observateurs de cette époque, met à part la fabrique de Clichy qui fournit en effet une quantité tout à fait exceptionnelle de malades : plus de $400 \%$ soit, par exemple, 209 malades pour 45 ouvriers en $1880^{15}$. En comparaison, Bezançon, qui emploie le même nombre d'ouvriers, envoie cette même année 15 ouvriers à l'hôpital. De plus, il est net que le nombre de malades chez Bezançon diminue significativement dans les années 1850 , ce que nous attribuons à des avancées techniques dans le mode de fabrication de la céruse; nous y reviendrons. Les données concernant les usines lilloises sont plus parcellaires. Le nombre d'entrées annuelles à l'hôpital n'excède pas 155 dans les années 1878-1880, pour plus de 300 ouvriers cérusiers. Il convient de manipuler ces chiffres avec prudence car, toujours d'après Gautier, beaucoup d'ouvriers habitent dans leur famille et s'y font soigner. Mais on peut tout de même distinguer la fabrique de Théodore Lefebvre, de loin la plus salubre de toutes avec de 4 à $6 \%$ d'ouvriers malades chaque année (seulement, a-t-on envie d'ajouter), et les autres, moins performantes en termes de salubrité, pour lesquelles il est compté de 20 à $50 \%$ de malades entre 1876 et 1879 (Gautier $1883: 253$; Desplats $\left.1878: 390^{16}\right)$. Là encore, nous attribuons cette différence à la qualité des opérations de la fabrication chez Théodore Lefebvre ${ }^{17}$.

Afin de montrer combien ils maîtrisent la situation sanitaire, et peut-être pour faire taire des rumeurs sur la faible espérance de vie de leurs ouvriers, les fabricants se félicitent d'avoir su les garder pendant longtemps. 10 des 91 employés de Théodore Lefebvre en 1852 ont 26 ans de maison (et 31 moins d'un an). Henri Leclerc, 
contremaître, et Jean-Baptiste Caby, employés par Théodore Lefebvre depuis 1825, sont encore présents en 1855 , soit 30 ans plus tard ${ }^{18}$. Bezançon souligne (1867:7) :

« Notre industrie, qui était autrefois à l'index et recrutait avec peine les bras qui lui sont nécessaires, trouve facilement aujourd'hui des ouvriers munis de livrets.

Plusieurs de nos hommes sont chez nous depuis 4, 6 et même 12 années. »

En 1900, Dubreucq donne le nom de quelques-uns de ses ouvriers : L. F., sujet belge, journalier depuis 20 ans en fabrique de céruse, dont 7 ans aux couches, 6 ans au broyage à l'huile, 3 ans aux ouvrages divers, depuis 4 ans contremaitre; W. I., journalier depuis 14 ans en fabrique de céruse, dont 1 an aux couches, et 13 ans au découchetage; V. H., belge, depuis 17 ans en fabrique de céruse; L.C., occupé en fabrique de céruse depuis 25 ans; M.C., depuis 19 ans en fabrique de céruse (Dubreucq 1900:15). Et Théodore Lefebvre termine ainsi la notice présentant sa fabrique à l'Exposition de 1889:

«Dévoués à leurs patrons, encouragés d'ailleurs par la certitude qu'une pension leur est allouée par nous lorsque l'âge les rend incapables de travailler, que leurs longs et bons services trouvent aussi leur consécration dans les récompenses publiques, ces modestes collaborateurs contribuent, par leurs connaissances pratiques, à la régularité de notre fabrication et au maintien de la réputation de notre marque. » (Lefebvre 1889)

Les améliorations apportées par les producteurs

16 Conscients de la dangerosité de la céruse et désireux de sauvegarder leur activité, les cérusiers traditionnels œuvrent pour rendre leur industrie plus salubre en introduisant de nombreuses innovations destinées à réduire la poussière émise. Pour comprendre les types de progrès réalisés, il est tout d'abord nécessaire de rappeler le mode de fabrication de la céruse. Le procédé traditionnel, importé en France depuis la Hollande, pays pour lequel son développement a été bien décrit (Homburg \& Vlieger 1996), autrement appelé procédé hollandais, est un procédé lent qui consiste à laisser des lames de plomb en contact avec du vinaigre et de l'acide carbonique généré par du fumier pendant environ deux mois. On obtient des écailles de céruse, qui sont ensuite broyées et vendues sous forme de pains.

Le plomb est importé, d'Espagne chez Delaunay à Tours ${ }^{19}$, d'Espagne ou d'Angleterre chez Théodore Lefebvre (Lefebvre et cie $1865: 29$ ), d'Allemagne chez la Vve Perus (Turgan $1885: 3$ ), sous la forme de saumons de 50 à $65 \mathrm{~kg}$ qui doivent être transformés, par fonte, en lamelles ou plaques fines avant d'être soumises à l'action du vinaigre. Les précautions à prendre sont celles en usage pour la fonte du plomb en général: il convient de diriger les vapeurs plombeuses vers une cheminée «ayant un tirage forcé ", selon les instructions du Conseil de Salubrité approuvées par la Préfecture de police de Paris en 1837 ou, après passage par une chambre de condensation, vers une cheminée "ayant au moins 10 mètres de hauteur », comme le demande le Préfet du Nord dans une directive de $1898^{20}$. Cette étape n'est pas la plus dangereuse et tous ne se conforment pas à ces instructions : ainsi Brabant, Bériot et Perus n'installent-ils pas de chambres de condensation, leurs cheminées étant respectivement de taille inférieure à $10 \mathrm{~m}$, supérieure de $10 \mathrm{~m}$ et de $20 \mathrm{~m}$ de haut en $1898^{21}$.

18 Les lamelles de plomb obtenues (typiquement de $0,5 \mathrm{~kg}$, de $60 \mathrm{~cm}$ de longueur et $10 \mathrm{~cm}$ de largeur) (Lefebvre 1889: 20) sont destinées à être enroulées sur elles-mêmes afin d'être placées dans les pots de terre où a lieu la réaction chimique de transformation du plomb en céruse. Les plaques fines présentent l'avantage d'une grande surface d'attaque (fig. 4a) mais, après la réaction, elles doivent être déroulées à la main afin 
d'en faire tomber la céruse qui s'est formée en surface. Cette opération dégage beaucoup de poussières car les plaques attaquées sont très fragiles et se cassent en plusieurs morceaux. Ces plaques ont été progressivement remplacées par des grilles, qui allient à une grande surface d'attaque une armature suffisamment épaisse pour qu'elle ne se brise pas lors de l'opération de décapage des écailles de céruse, le plomb réagissant sur une épaisseur de 3 à 4 mm (fig. 4b) (Bouchonnet 1909 : 96).

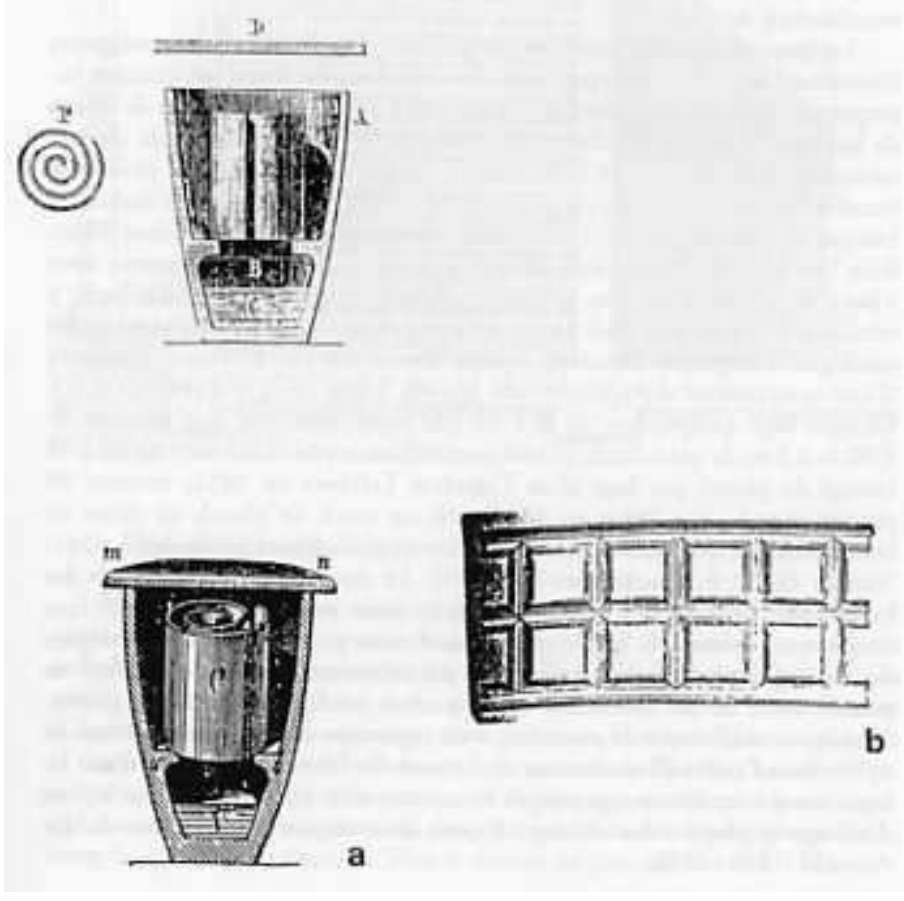

Figure 4 a) Pot à céruse avec spirale de plomb ; b) grille

Les gravures proviennent de M. J. Girardin (1869), sauf la figure 4 b (Troust 1894)

Les grilles ne sont alors plus introduites dans les pots, mais simplement posées dessus. En fin de décapage, ces grilles facilement manipulables sont renvoyées en fonderie. Mais, dans les années 1870, les deux leaders dans l'amélioration des procédés que sont Bezançon à Paris et Th. Lefebvre à Lille, utilisent toujours des lames de plomb. En 1881, il est recommandé à Bériot, lors de l'installation de son usine, de remplacer les lames de plomb par des grilles rectangulaires, forme que l'on retrouve chez la Vve Perus en 1885 (Freycinet 1870: 73; Turgan 1885: 3). Toutes les descriptions du XXe siècle mentionnent des grilles ${ }^{22}$.

Les pots, chargés de lames ou des grilles de plomb et d'acide pyroligneux (concernant de l'acide acétique) sont installés dans des loges, en couches superposées, chacune composée d'une série de 1000 à 1500 pots, et de $40 \mathrm{~cm}$ de hauteur de fumier de cheval, assurant à la fois le dégagement de chaleur nécessaire à la réaction et la formation du gaz carbonique qui permet la transformation de l'acétate de plomb, à la surface des plaques, en carbonate basique de plomb (fig. 5). Les ouvriers travaillent au montage (chez Théodore Lefebvre, 4 ouvriers sont affectés à ce poste en 1852 et montrent deux « lits » ou couches en une journée, Lefebvre et Cie $1865: 161$ ), puis, 6 semaines de 2 mois plus tard, temps nécessaire à la réaction, au démontage de ces loges. Les grands cérusiers, comme Bériot ou Th. Lefebvre, disposent d'une quarantaine d'emplacements appelés loges, la Vve Perus en a 65. Chaque loge comportant de 8 à 10 lits superposés, 
sur une hauteur de $4,50 \mathrm{~m}$ à $5 \mathrm{~m}$, la quantité de plomb immobilisée est considérable : de 10 à 12 tonnes de plomb par loge chez Théodore Lefebvre en 1852, environ 40 tonnes chez la Vve Perus en 1885, soit un stock de plomb en cours de transformation qui peut dépasser 1500 tonnes (Lefebvre et Cie 1865:161; Turgan 1885: 4; Bouchonnet 1909: 94). Le montage et le démontage des loges sont considérés comme des opérations peu dangereuses. Il faut simplement veiller à la bonne qualité du fumier pour éviter qu'il ne dégage de l'anhydride sulfureux transformant partiellement la céruse en sulfure de plomb, noir, ce qui conduirait à des qualités médiocres de céruse, grisées. Pour éviter tout risque de poussière, il est cependant recommandé à Bériot en 1881, dans l'arrêté d'autorisation de sa nouvelle fabrique, d'arroser d'eau les loges avant tout démontage jusqu'à humectation de la céruse, comme le font d'ailleurs la plupart des cérusiers à cette date, depuis l'exhortation du Dr Arnould (1878: 638).

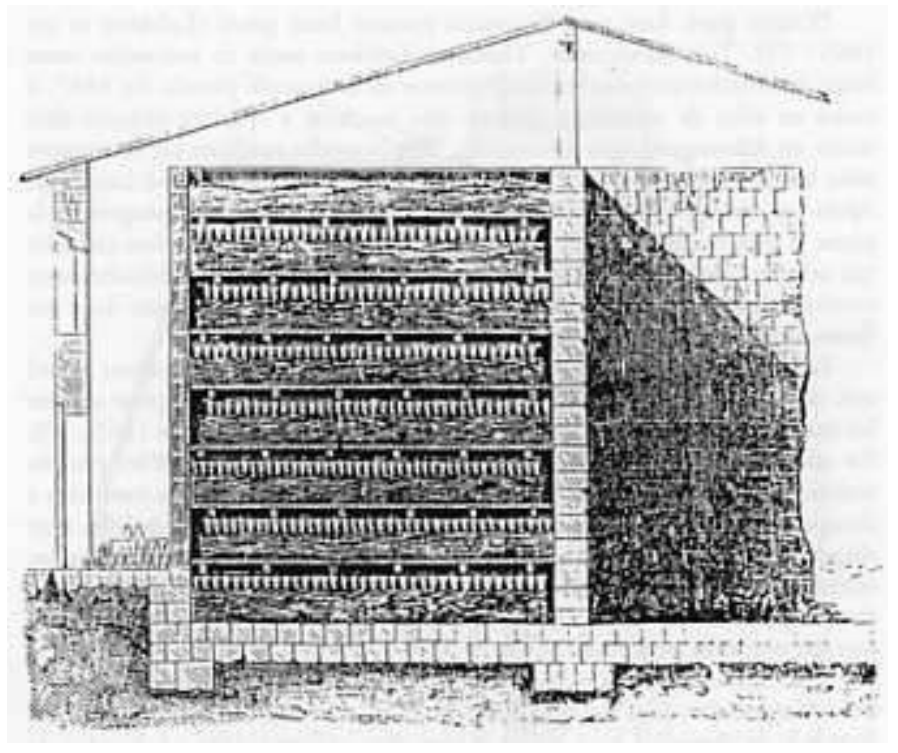

Figure 5. Loges permettant la fabrication de la céruse par le procédé hollandais

Après le démontage, vient le décapage des lames carbonatées. Cette opération est reconnue comme la plus dangereuse de toutes. La première technique consiste à détacher les écailles de céruse de la lame de plomb à la main, par battage des lames avec un maillet en bois. Il est conseillé tout d'abord d'effectuer cette opération dans un atelier bien ventilé ou employant le tirage de la cheminée. C'est ensuite la protection de l'ouvrier lui-même qui est préconisée, par le port de gants et de masques : par exemple un «masque qui couvre la moitié inférieure du visage et qui est formé d'une éponge serrée entre deux toiles métalliques ». Mais le remède est pire que le mal :

«Le principal défaut de cet appareil, c'est qu'il a besoin, d'être savonné tous les jours. En outre, l'interposition de l'éponge le rend très échauffant, au point que les ouvriers le soulèvent de temps en temps pour respirer plus librement. Aussi, chez M. Orsat, fabricant de céruse, à Clichy, les ouvriers ont-ils renoncé à s'en servir, parce que c'était pour eux une occasion de porter perpétuellement au visage leurs doigts chargés de matière toxique (Freycinet $1870: 49$ ).

D'autre part, bien peu d'ouvriers portent leurs gants (Lefebvre et Cie $1865: 59$ ). Très rapidement, Théodore Lefebvre tente de mécaniser cette étape de détachement des écailles de céruse de la lame de plomb. En 1837, il essaie en effet de mettre en marche une machine à cylindre cannelé déjà usitée en Allemagne, mais sans succès. Une 
nouvelle machine est finalement mise au point en 1842, par le menuisier-mécanicien lillois Aimé Laurenge. Après un premier grattage manuel qui permet de dépouiller les plaques de la partie la plus friable, celles-ci sont ensuite passées entre des cylindres cannelés qui achèvent de les décaper (fg. 6). Le blanc en écaille qui s'en détache passe entre de nouveaux jeux de cylindres et tombe à travers un blutoir dans des fosses souterraines (fig. 7).

Laurenge est rapidement sollicité par d'autres producteurs, comme Roard qui, dès le 2 mai 1842, lui demande le prix de sa «mécanique pour séparer les écailles des lames de plomb sortant des loges» (Lefebvre et Cie 1865 : 59). En effet, Théodore Lefebvre communique aux autres fabricants les progrès techniques réalisés dans son usine, et notamment les plans de ces machines à décaper les lames de plomb et à pulvériser la céruse qui, "outre qu'elles font disparaitre les opérations manuelles les plus dangereuses de la fabrication, réalisent une importante économie de main-d'œuvre ». L'opération qui exigeait le concours de 10 ouvriers chez Guichard à Nantes, n'est plus dirigée que par une seule personne ${ }^{23}$.

Mais ces machines ne constituent pas une panacée. Les opérations ont beau être menées dans des appareils "parfaitement clos", "hermétiquement fermés", le dispositif tout entier a beau être enfermé dans une espèce de grande armoire à double ou triple porte, rien ne semble pouvoir arrêter la poussière. Les ouvriers appellent la machine à décaper "le diable » en raison des «allures bruyantes et farouches, mais aussi pour sa malignité réelle; un appareil à mouvements violents comme celui-là est fatalement un générateur de poussière " (Arnould 1878 : 640). Pour récupérer la céruse, il faut interrompre le travail pendant un certain temps, 24 heures au moins dit Freycinet (1870: 73), afin de lui laisser le temps de se déposer (cf. aussi Tardieu 1862 : 342). Ces machines sont alors progressivement abandonnées et le décapage redevient une opération manuelle, à des dates variées selon les producteurs; 1867 chez Théodore Lefebvre, 1871 chez Besançon, 1881 chez Bériot, mais seulement en 1894 chez la Vve Perus, qui avait breveté un appareil de décapage à sec en 1884, fonctionnant grâce à des martelets mus mécaniquement (Arnould 1879 : 4; Turgan $1885: 5$ 5 $^{24}$.

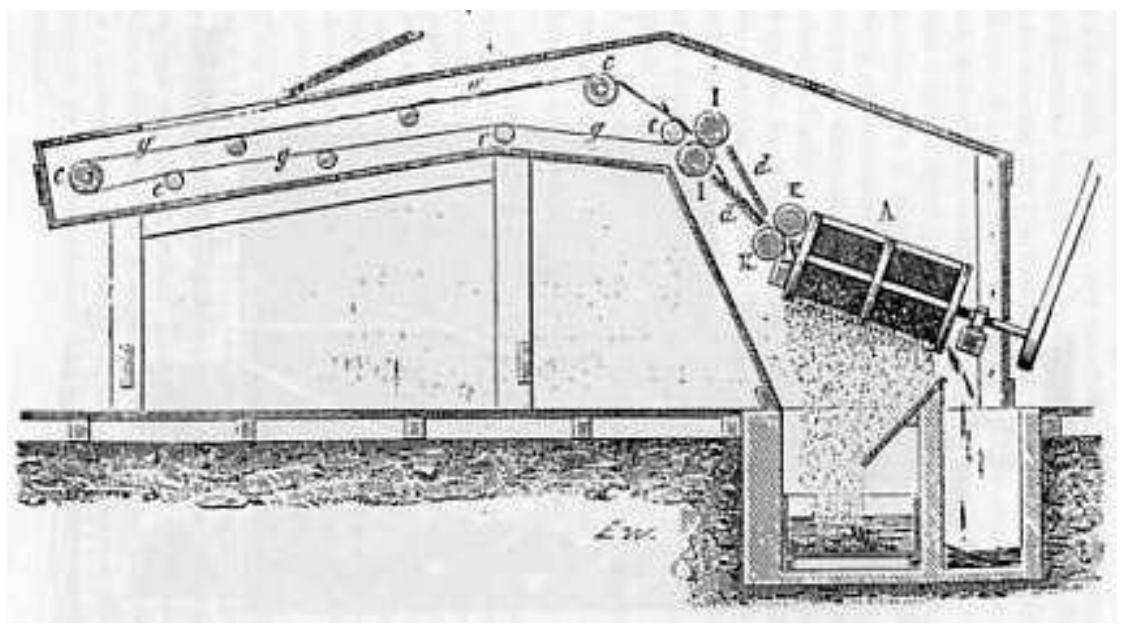




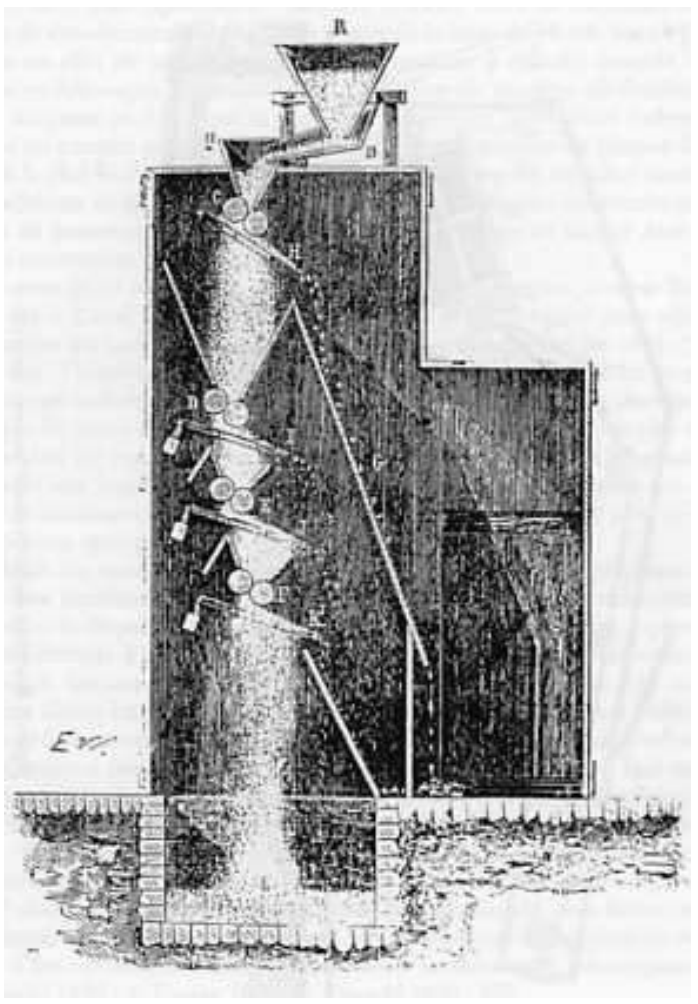

Figure 7. Machine à écraser la céruse

Cependant, ce décapage n'a plus lieu à sec mais sur de la céruse mouillée ou humectée. Non seulement l'usage de l'eau permet d'éviter la formation de poussière, mais l'arrosage des couches avant leur démontage provoque le refroidissement brusque du plomb qui passe de 50 ou $60^{\circ} \mathrm{C}$ (température dans les couches due à la fermentation du fumier) à la température ambiante, cela conduisant à la rétractation du plomb : «il en résulte que les écailles se fendillent, se soulèvent, se détachent du plomb qui se retire de son côté ". Il suffit alors "de très légers coups de maillet pour faire tomber ces écailles qui ne demandent qu'à se détacher" (Arnould 1878: 639). En 1898, la Préfecture du Nord recommande le décapage manuel sur de la céruse humectée mais ne rejette pas le décapage mécanique, à condition qu'il ait lieu dans des enceintes closes à l'aide d'une double enveloppe complètement hermétique. Ce n'est qu'avec les progrès des moyens de ventilation qu'Expert-Bezançon envisagera d'installer à nouveau les machines à décaper dans sa nouvelle usine d'André-lez-Lille. Les grilles y sont tout d'abord humectées avant d'être écrasées dans des cylindres cannelés en bronze surmontés d'une très bonne aspiration (Bouchonnet 1909: 98).

Une étape importante est le remplacement du broyage de la céruse dans des meules verticales par des meules horizontales ${ }^{25}$. Cette innovation est introduite en 1849 par le producteur lillois Théodore Lefebvre:

« Au lieu des meules verticales fonctionnant à air libre, M. Lefebvre a adopté les meules horizontales parfaitement closes, la supérieure seule étant rendue mobile par un arbre vertical; une boîte hermétiquement fermée enveloppe les deux meules, de sorte que la pulvérisation se fait à vase clos et que la poussière ne peut se répandre dans l'air. Une calotte en cuivre, fixe à l'aide de vis au tambour de la boîte, en ferme le dessus, et c'est sur cette calotte qu'est établi le distributeur, que, 
pour éviter une longue description, nous comparerons à un grand moulin à café qui reçoit la céruse, la concasse et la laisse passer dans les meules, après l'avoir pulvérisée, la poussent dans des blutoirs aussi hermétiquement fermés que les meules. » (Bailly 1849 : 4)

Dans cette première description, on constate que le broyage a encore lieu à sec, mais qu'est mis en place l'entourage des meules par des garnitures en cuivre, difficiles à installer autour de meules verticales. Pourtant, le broyage à l'eau était à cette date déjà connu et décrit chez Mouvet et Mathieu d'Orléans dès $1823^{26}$. Il est donc très vite appliqué à ces meules horizontales qui s'y prêtent bien. L'entourage des meules permet en effet d'éviter les éclaboussures de céruse mouillée sur les ouvriers. Les effets positifs sont immédiats :

«Depuis dix-huit mois que l'on opère le battage des couches sous l'eau, qu'on passe de suite la céruse aux meules humides, sans l'écraser préalablement à l'état sec, et qu'on nettoie les pots après les avoir fait séjourner plusieurs jours sous l'eau, on ne voit pas moitié autant d'ouvriers atteints de maladie saturnines que par le passé. » (Lefebvre et Cie $1865:$ 48)

De manière générale, les producteurs de céruse vont ainsi tenter de profiter des bienfaits de l'eau à toutes les étapes. L'intérêt de l'eau a semble-t-il été souligné par Roard et son contremaître Bergerat, qualifié de chimiste fort instruit :

«Ces observateurs ont été conduits à employer l'eau comme moyen préservatif, par ce qu'ils avaient appris au sujet des fabriques de céruses anglaises. En effet, dans ce pays toutes les opérations se font presque à l'eau, et l'on assure qu'il y a beaucoup moins d'ouvriers atteints de maladies saturnines dans ces établissements qu'en France. » (Lefebvre et Cie $1865: 48$ )

Cette vision est soutenue par le Dr Arnould, médecin à Lille :

«Le moyen d'assainir considérablement, sinon d'une façon complète l'industrie de la céruse, c'est, depuis le moment où elle est naissante et enfermée dans les fosses jusques et y compris le moment où elle arrive au peintre, de ne travailler jamais la céruse qu'à l'état mouillé. " ${ }^{27}$

Il s'agit cependant d'éviter que les ouvriers ne pataugent maintenant dans une eau chargée en céruse. Se pose en effet la question du passage transcutané de la céruse :

«Le contact des mains avec l'eau laiteuse tenant en suspension le carbonate de plomb, le toucher des pâtes, des ustensiles, blutoirs à ouvrir, à fermer, truelle, colligettes, etc., contact des vêtements mouillés ou tachés de ces mêmes matières, fournissent autant de causes d'absorption saturnines par la peau et dans les poils: nous en avons d'intéressants exemples dans la thèse du docteur Samsoen de Lille. " (Loir, Ferrand \& Radisson $1883: 8$ )

31 Cette constatation conduit les producteurs à contrôler la quantité d'eau utilisée : les ouvriers qui, dans un premier temps, inondaient les loges avant de procéder à leur démontage, vont rapidement revenir à une simple humectation des couches de fumier. De même pour les étapes suivantes, où la céruse est simplement humectée lors du décapage, puis lors du broyage. Millot-Cousin (1878: 7) peut ainsi se féliciter de ce que, depuis 1872, aucun ouvrier n'est sorti de sa fabrique " pour cause de maladie provenant de la respiration des poussières, ni même de leur absorption par les pores de la peau ».

Après le broyage à l'eau, la céruse est mise dans des pots en cuivre qui contiennent $1,5 \mathrm{~kg}$ environ et sont placés dans des séchoirs jusqu'à l'obtention de pains secs. Ces derniers sont enveloppés dans du papier et vendus ainsi; ou bien la céruse est pulvérisée dans un moulin à noix et embarillée dans des tonneaux puis tassée grâce à un disque en bois sur lequel agit une presse. Il est fortement conseillé de procéder à cet 
embarillage par des procédés mécaniques, mais en 1893, la Vve Perus renonce à ce procédé faute de résultats satisfaisants et ses ouvriers chargent les tonneaux avec des pelles, sans précautions particulières (fig. 8) ${ }^{28}$. Cependant, les producteurs, et notamment Bezançon à Paris, ont tenté de promouvoir dès 1845 la vente de céruse sous forme de pâte à l'huile, tout d'abord obtenue par empâtage de la céruse en poudre dans des malaxeurs. Puis, profitant de la meilleure affinité de la céruse pour l'huile que pour l'eau, particularité signalée par Clément-Desormes dès 1834, cet empâtage est réalisé sur de la céruse humide, introduite dans des pétrins mécaniques, avec une quantité convenable (8 à $10 \%$ ) d'un mélange formé d'un tiers d'huile de lin et deux tiers d'huile d'œillette (Lefebvre et Cie $1865: 9)$, Freycinet $1870:$ 74). L'huile se substitue entièrement à l'eau qui est enlevée par simple décantation. La pâte ainsi obtenue est passée entre deux ou plusieurs broyeurs à cylindres jusqu'à obtention de l'onctuosité voulue (fig. 9). Bezançon semble avoir cessé complètement la fabrication de céruse en pain et en poudre au profit de la céruse à l'huile dès 1852 (1867: 5). Lefebvre adopte le procédé vers 1850. Il installe trois broyeurs à quatre cylindres en 1866 et met au point la même année un broyeur à cinq cylindres, mais n'abandonne pas pour autant la production de céruse en poudre. En 1870, il vend la moitié de sa céruse en poudre, l'autre moitié en pâte, à l'eau ou à l'huile. En 1881, la fabrique Lefebvre produit encore un peu de céruse en poudre, par égard pour quelques clients. En 1917, cette usine a en stock 88 tonnes de céruse broyée à l'eau, 81 tonnes de céruse broyée à l'huile et 4 tonnes de céruse en poudre ${ }^{29}$.

On constate ainsi que la plupart des avancées techniques ont été introduites par deux fabricants qui jouent le rôle de meneurs: Bezançon à Paris, et Théodore Lefebvre à Lille. Ils appliquent ces innovations à leurs fabriques avec rigueur, et contribuent ainsi à abaisser le taux de malades dans leurs entreprises. Outre ces changements techniques, de nombreuses consignes de bon sens ont été imposées par des arrêtés préfectoraux édictés lors de l'ouverture d'une fabrique ou suite aux enquêtes des inspecteurs de salubrité de Paris ou de Lille. Il est ainsi recommandé de laver les murs à grande eau, de ne pas balayer le sol à sec, de mettre en place un vestiaire où les ouvriers puissent se laver les mains et se changer avant de quitter leur lieu de travail, d'interdire de manger dans les ateliers. 


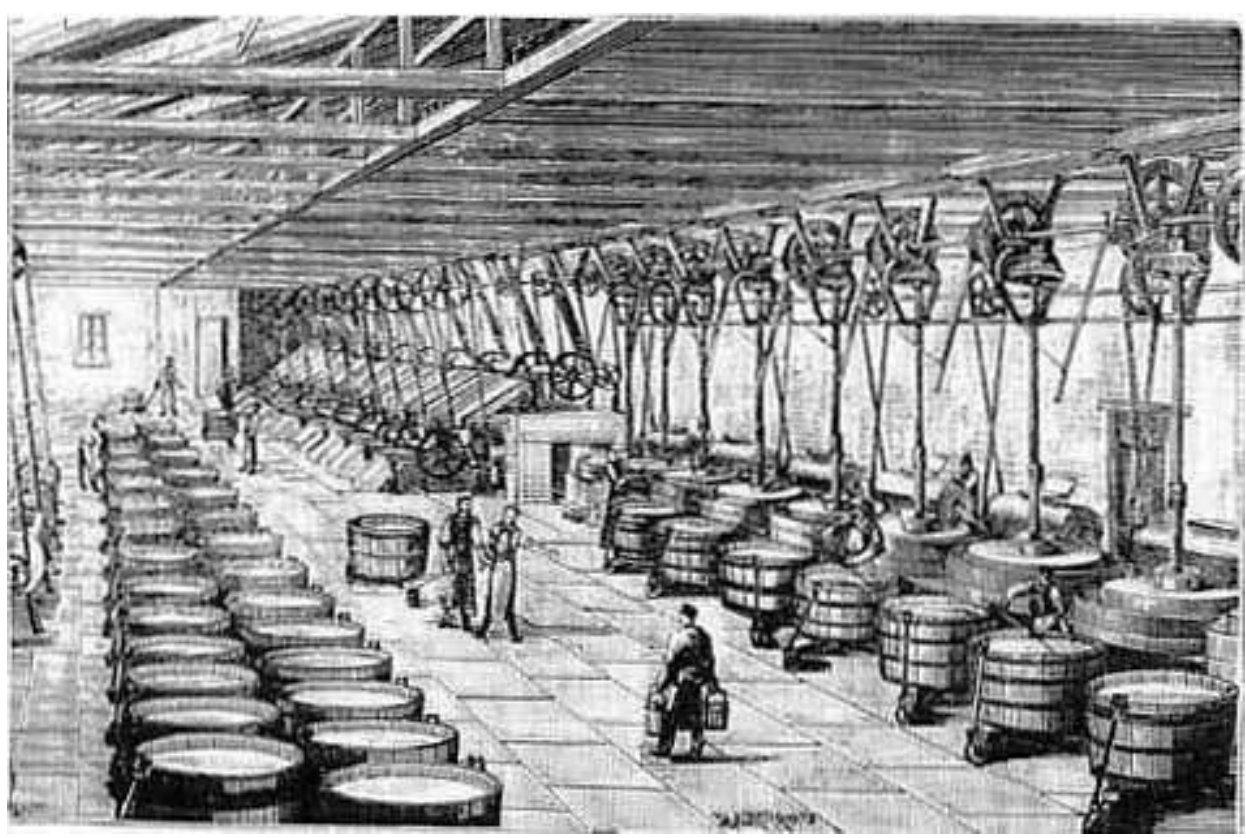

Figure 8. Atelier de Broyage chez la Vve Perus à Lille (Turgan 1885)

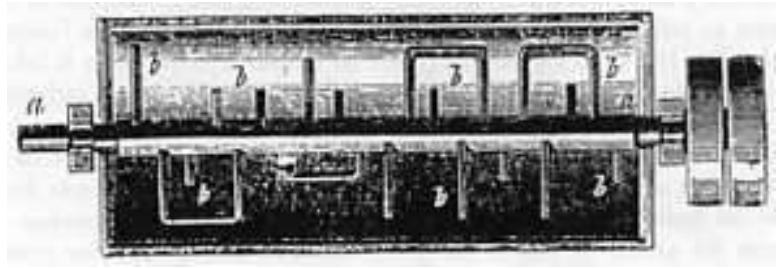

Figure 9. Cylindre à empâter d'huile la céruse broyée à l'eau

Les procès verbaux de récolement de l'arrondissement de Lille des années 1893 et 1898 montrent que ces consignes sont rarement prises en compte, ce qui contribue directement au mauvais état sanitaire des ouvriers ${ }^{30}$. Ce n'est pas le cas chez Bezançon :

«L'ouvrier est tenu, chaque fois qu'il quitte son travail, de se laver dans des baquets contenant une solution faible de sulfure de potassium. Ces baquets sont disposés extérieurement, le long des murs de l'atelier et sur le passage des hommes, de manière à ce que ceux-ci ne puissent éviter de les rencontrer. » (Freycinet $1870: 60$ )

Par ailleurs, certains producteurs ont mis eux-mêmes en place des procédures particulières avec un certain effet positif. La plus suivie est le fait d'établir un roulement des ouvriers aux postes dangereux.

"Il s'agit de ne pas laisser indéfiniment les mêmes ouvriers aux travaux dangereux, mais au contraire de les faire alterner avec ceux qui sont occupés aux autres branches de la fabrication, de manière à ce que les uns et les autres restent chaque fois exposés pendant une période de temps assez courte. » (Freycinet 1870 : 61)

Cependant, une usine semble ne pas suivre le mouvement général et envoie, pendant toute la durée de son existence, des centaines de malades à l'hôpital chaque année. On peut, à juste titre, se demander si cette situation est liée au procédé de fabrication employé, le procédé de Clichy, ou à une gestion particulière de ce site.

Le cas particulier de Clichy 

d'un procédé innovant, qu'a proposé le chimiste Thénard, et dont la mise au point industrielle a été réalisée par Roard, directeur de l'usine de Clichy (fig. 10). C'est un procédé rapide en milieux aqueux, où de la litharge (oxyde de plomb) est mise à réagir avec $d u$ vinaigre, puis du gaz carbonique qui barbotte dans la solution par l'intermédiaire de centaines de tubes.

Ce procédé permet d'obtenir en moins de 48 heures une céruse de très belle qualité sous la forme d'une poudre finement divisée, mais très dangereuse car facilement inhalée par les ouvriers. L'usine de Clichy produit également des oxydes de plomb (massicot, minium, litharge et mine orange), qui, eux aussi, peuvent être responsables d'« une grande proportion des malades qui sortent de cette maison» (Gautier 1883 : 247). Mais ces deux particularités ne peuvent à elles seules expliquer le nombre élevé de malades provenant de cette usine. En effet, l'usine de Clichy est infiniment plus dangereuse que celle de Portillon, près de Tours, où l'on fabrique aussi de la céruse par le procédé de Clichy, du minium et du massicot. « À Portillon, en moyenne 10 entrées à l'hôpital pour 100 ouvriers. À Clichy, 400 entrées pour le même chiffre » en 1878 (Gautier 1883: 248). D'autres fabriques de minium, comme celle de Baccarat, ont semble-t-il réussi à rendre leurs ateliers moins insalubres, avec moins de $5 \%$ de malades (Gautier 1883: 251; Dogat-Moriceau 1998). De plus, à une date que nous n'avons pas identifiée mais au plus tard en 1862, l'usine de Clichy abandonne même le procédé de Thenard au profit du procédé hollandais (Tardieu 1862 : 341). En fait, la littérature abonde sur les raisons pour lesquelles cette usine s'avère si dangereuse : Roard, puis Orsat, son gendre et successeur, et enfin le fils de ce dernier, se sont fait une spécificité de la céruse en poudre, la meilleure selon eux produire de la mine orange (l'oxyde de plomb incorporé dans le cristal de luxe) par calcination. Ainsi Roard est-il le seul fournisseur en oxyde de plomb de la cristallerie de Saint-Louis en $1844^{31}$. Tout est dit.

« Dans l'usine de Portillon, la céruse est fabriquée par la méthode Thénard sans jamais passer à l'état de poudre sèche; le défournement et le broiement du massicot et du minium sont faits avec les grandes précautions et en vases clos autant que possible. » (Gautier 1883 : 248)

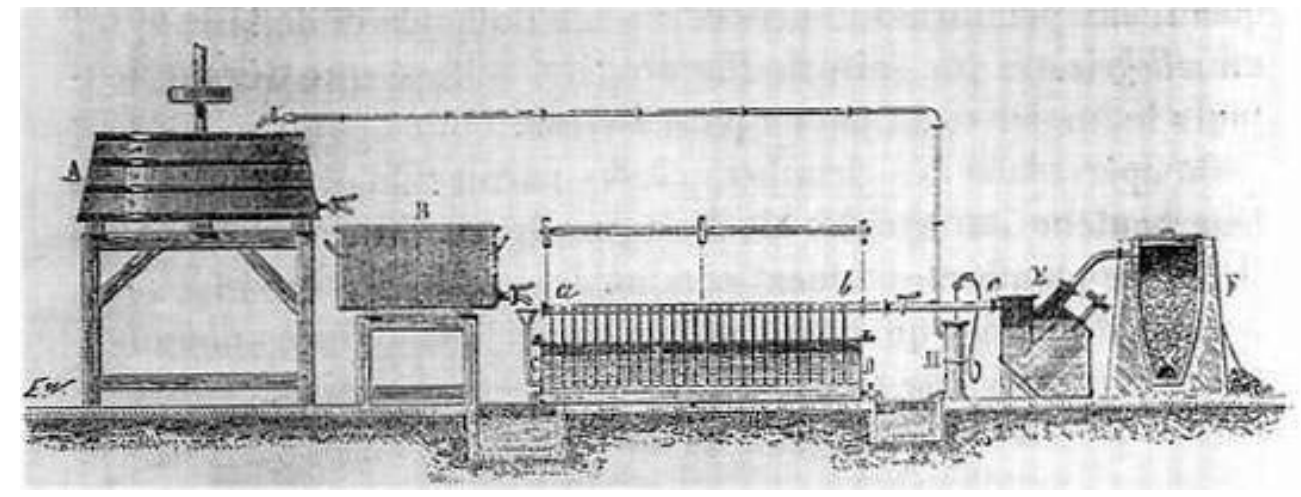

Figure 10. Production de céruse par le procédé de Clichy

39 À Clichy, au contraire, la céruse est recueillie, battue, et moulue à l'état sec avec production d'abondantes poussières; des moulins et des broyeurs de céruse et de minium s'élève " un incessant nuage plombifère » (Gautier 1883: 248). Roard était, d'après Théodore Lefebvre, à l'origine de l'utilisation de l'eau pour réduire les 
poussières lors des étapes de décapage et de broyage de la céruse, mais son gendre n'applique manifestement pas ses recommandations: en 1877, les opérations d'épluchage et de décapage sont faites « non seulement à la main, mais à nu par simple battage comme on l'a pratiqué à l'origine de la fabrication ».Alors que tous les fabricants adoptent le principe du broyage à l'eau de la céruse, à Clichy, au contraire, "la céruse séchée à l'étuve est réduite en poudre sous des meules installées dans un atelier assez mal aéré, avec un appareil complètement ouvert. Les ouvriers apportent dans des caisses en bois la céruse sèche très friable et la jettent sur la table des meules où elle doit être broyée, ils la recueillent écrasée et tombant en poudre de cette table pour aller la verser dans un blutoir voisin. Le travail soulève de la poussière de céruse qui recouvre les murs, les charpentes et tous les objets apparents de l'atelier $»^{32}$. Cette situation justifie amplement le nombre de malades provenant de cette fabrique, nombre qui chute évidemment à la fermeture de l'usine en 1882 .

La gestion de la toxicité de la céruse par l'État

La première intervention de l'État est une loi royale promulguée en 1823, mettant en demeure les cérusiers de vendre exclusivement de la céruse en poudre (la forme la plus dangereuse) et interdisant la vente de la céruse en pains. Cet épisode illustre la manière dont le gouvernement français s'informe et prend des décisions. Lorsqu'il est saisi par la direction des hôpitaux du grand nombre de malades de saturnisme qui se font hospitaliser au début des années 1820, le ministre de l'Intérieur se tourne vers le Comité Consultatif des Arts et Manufactures, composé d'experts qui, après étude du dossier technique, rendent leur avis. Or, ce Comité est composé, à cette date, de chimistes très liés à l'usine de Clichy: Thénard -à l'origine du procédé-, Gay-Lussac, collègue de Thénard, et Roard, directeur de l'usine. Ceux-ci défendent leur procédé original et tentent de faire admettre que c'est la manipulation de la céruse en poudre fine pour la mettre en pains, forme sous laquelle est vendue la céruse du procédé hollandais, qui induit la maladie. Les cérusiers lillois s'élèvent à juste titre contre cette loi qui les oblige à broyer les pains de céruse en poudre fine, éminemment plus dangereuse. La loi est donc abrogée en 1825 (AN F12 2428).

L'État intervient par l'intermédiaire des membres du Conseil de salubrité du département de la Seine, notamment Alphonse Chevallier qui visite les fabriques, écrit des rapports et propose des instructions relatives aux précautions à prendre lors de la fabrication de la céruse. Si les producteurs lillois sont les pionniers pour les innovations tendant à réduire la dangerosité de la fabrication, c'est la préfecture de la Seine qui, la première, se préoccupe d'établir des instructions relatives aux précautions à prendre. Ces instructions, datant de 1834 et de 1837, s'inspirent directement des progrès réalisés chez le producteur lillois Théodore Lefebvre qui a reçu pour l'occasion une délégation d'enquêteurs parisiens ${ }^{33}$.

L'état tente en 1848 de favoriser le remplacement de la céruse par un produit de substitution, lors d'une campagne "prescrivant la substitution du blanc de zinc au blanc de céruse dans les travaux de peinture à exécuter dans les bâtiments de l'État "; sans succès, car les ouvriers peintres reprochent aux peintures à base de cet oxyde de zinc leur mauvais pouvoir couvrant et leur moins bonne tenue sur les murs.

La question de la toxicité du procédé de fabrication de la céruse est largement présentée par le Dr Arnould lors du Congrès International d'Hygiène (1878: 638-642), qui se tient à Paris en août 1878, en marge de l'Exposition Universelle. Ce congrès est présidé par le Dr Gubler, médecin depuis 24 ans à l'hôpital Beaujon, qui accueille la 
majorité des malades de l'usine de Clichy. Est-ce suite à ce congrès que le département de la Seine produit à nouveau, en 1881, une instruction concernant cette fois-ci la fabrication et la manipulation du plomb et de ses dérivés, dont la céruse n'est qu'un exemple $^{34}$ ? C'est à la suite d'un rapport de l'inspecteur de l'insalubrité de la Préfecture du Nord du 27 mars 1898, constatant le grand nombre de saturnins qui se trouvent en traitement à l'hôpital Saint-Sauveur, que le préfet du Nord prend un arrêté en date du 10 juin 1898 pour réglementer à nouveau l'exploitation des fabriques de céruses à Lille, ce qui permet d'établir un état des lieux des instructions prescrites et de la manière dont elles sont traduites dans les fabriques lilloises, systématiquement contrôlées par les inspecteurs de salubrité si le nombre de malades provenant de ces fabriques est trop élevé (AD59 M417 5089).

Il faut attendre un intense campagne de presse menée dans l'Aurore en 1901, constatant que "voici cinquante ans que l'État ne fait rien contre l'emploi de la céruse " (cité par Viet 1994: 285), pour qu'apparaisse le premier décret qui en réglemente l'usage en 1902, où il est prescrit que la céruse doit obligatoirement être employée sous forme de pâte dans l'industrie de la peinture en bâtiment ${ }^{35}$. Puis la loi du 20 juillet 1909 limite, non pas la fabrication de la céruse, mais son usage « dans tous les travaux de peinture de quelque nature qu'ils soient, exécutés par les ouvriers peintres, tant à l'intérieur qu'à l'extérieur des bâtiments ». Cette loi est censée entrer en application en 1915, mais la production de céruse, interrompue pendant la Première Guerre mondiale (la plupart des cérusiers étant installés dans la région lilloise), reprend ensuite pour atteindre en 1920 des tonnages identiques à ceux d'avant-guerre (AD59 10R 5099). Ces dispositions, finalement appliquées dans les années 1920, interdisent aux ouvriers peintres en bâtiment l'usage de peinture à base de céruse, mais on constate qu'elles ne protègent pas les artisans indépendants,ce que corrigera la loi de 1926, interdisant l'emploi de la céruse pour tous les professionnels du bâtiment. Les autres usagers de la céruse, pourtant recensés par Layet en 1894 (artistes peintres, peintres de décors, menuisiers pratiquant le cérusage du bois, ébénistes, serruriers et vitriers qui rabotent pour les besoins de leur profession les plinthes, bois de fenêtres ou de portes, blanchisseurs de dentelles, fabricants de cosmétiques et parfumeurs) peuvent continuer à utiliser la céruse sans restriction. Ne sont pas non plus concernés les vernisseurs qui emploient des vernis à base de céruse pour le glaçage des poteries et des porcelaines, la fabrication de cuirs vernis, de toiles cirées ou de cartes de visite vernissées $\mathrm{Ni}$ les usagers de mastics au plomb, à base de céruse. Ni, enfin, les victimes " passives » de ces usages (boulangers utilisant du bois de récupération anciennement peint à la céruse ${ }^{36}$ et blanchisseurs de linge, sans compter tous les particuliers emménageant dans des logements dont les peintures viennent juste d'être poncées), dont personne ne semble se soucier.

Enfin, la production de céruse n'est pas interdite. De nouvelles autorisations pour la fabrication de minium, litharge et céruse sont même accordées à l'entreprise Villemot d'Aubervilliers le 10 mars 1926, ou à Expert-Bezançon de St André-lez-Lille le 19 mai $1930^{37}$. On constate toutefois une diminution de la production française, tandis que les industriels lillois se convertissent à la production de blanc de zinc, puis passent à la simple formulation de peintures lorsqu'arrive sur le marché un produit de substitution accepté par tous : l'oxyde de titane, à partir de 1922.

Alors que la fabrication de céruse est reconnue comme un métier dangereux dès ses débuts, cette industrie se développe en France au XIXe siècle, poussée par une demande 
croissante en murs blancs. La production industrielle se développe d'abord à Clichy, puis se concentre dans la région Nord, où se situe le cérusier le plus innovant: Théodore Lefebvre. Les grands cérusiers, conscients qu'il faut améliorer leur procédé pour remédier au sort de leurs ouvriers, atteints de saturnisme, montrent une bonne volonté reconnue par tous : innovations techniques, instructions destinées à protéger les ouvriers contre les poussières de céruse, masquant par leurs bons résultats la réalité d'autres fabriques moins salubres. Le cas extrême en est représenté par l'usine de Clichy, non pas du fait de son procédé de fabrication particulier, mais parce qu'elle manipule la céruse à sec et refuse de l'humidifier lors de son traitement. La proportion de malades de saturnisme provenant des fabriques de céruse est considérable, mais ce secteur d'activité n'employant finalement que peu d'ouvriers, ce sont les peintres qui représentent à la fin du XIXe siècle la plus grande part des malades. Cela peut expliquer le fait que c'est l'usage de la céruse, et non pas sa fabrication, qui est finalement interdit. Ses méfaits sont suffisamment graves pour inspirer le poème suivant :

"Que de gens, à trente ans, sont déjà des vieillards

Pour avoir respiré le blanc poison qui vole

Dans la lumière, avec le souffle et la parole !»

Hugues (1929) ${ }^{38}$

\section{BIBLIOGRAPHIE}

Arnould (Dr), Jules

1879.Assainissement de l'industrie de la céruse. Société industrielle du Nord de la France. L.

Danel.

Bailly, aîné

1849.«Fabriques de céruse. Rapport sur l'état hygiénique des fabriques de MM. Th. Lefebvre et Poelman frères, à Moulins-Lille ", Comité central d'hygiène et de salubrité du département du nord. Lille.

Bezançon, Frères

1867.Note présentée par MM Bezançon Frères, fabricants de céruse à Paris, Classe 44 - nº 91 à la commission impériale de l'Exposition de 1867. Paris : Renou et Maulde.

Bouchonnet, A.

1909.Industries du plomb et du mercure. Paris : O. Douin et Fils (tome 2).

Chevallier, Alphonse

1849.Sur la substitution du blanc de zinc et des couleurs à base de zinc au blanc de plomb et aux couleurs à base de plomb et de cuivre, par M. Leclaire, entrepreneur de peintures. Paris.

Desplats, Henri

1878. « Histoire sanitaire des fabriques de céruse à Lille, depuis 1866 jusqu'à 1878 ", Annales

d'Hygiène publique et de médecine légale 47 : 385-406.

Dubreucq, Horace

1900.Monographie d'une industrie insalubre, Exposition Universelle de 1900. Lille : L. Danel. 
Dogat-Moriceau, Caroline

1998.« La politique sanitaire à la Compagnie des Cristalleries de Baccarat (1850-1914) », pp. 51-62, in De la verrerie forestière à la verrerie industrielle. Aix-en-Provence : Danielle Foy Éditions.

Engel, Gustave

1879.« Les produits chimiques à l'Exposition Universelle de Paris en 1878, examinés plus spécialement au point de vue de l'industrie des toiles peintes ", Bulletin de la Société Industrielle de Mulhouse 49 : 53-83.

Faure, $\mathrm{H}$.

1889.Histoire de la céruse. Lille : Imprimerie Lefèbre-Ducrocq.

Freycinet, Charles (de)

1870.Traité d'assainissement industriel comprenant la description des principaux procédés employés dans les centres manufacturiers de l'Europe occidentale pour protéger la santé publique et l'agriculture contre les effets des travaux industriels. Paris : Dunod.

Gautier, Armand E-J

1883.Le Cuivre et le plomb dans l'alimentation et l'industrie au point de vue de l'hygiène. Paris : J. B.

Baillère et fils.

Girardin, Jean P. L.

1869.Chimie générale et appliquée (4ème année). Paris : Victor Masson \& fils.

Héricart de Thury, Louis E.-F. et Migneron

1827.Rapport sur les produits de l'industrie française.

Homburg, Ernst \& Johan H. de Vlieger

1996. « A victory of practice over science : the unsucessful modernization of the dutch white lead industry (1780-1865) », History and Technology 13 :33-52.

Hugues, Clovis

1929.L'empoisonné. Paris : Les Bonnes Lectures.

Kuhlmann, Frédéric

1834.Rapport du jury départemental du Nord sur les produits de l'industrie admis au concours de l'exposition publique de 1834. Lille : L. Danel.

1849.Exposition nationale des produits de l'industrie agricole et manufacturière de 1849. Rapport du jury départemental du Nord: analyse de la situation industrielle du département. Lille : L. Danel.

Layet, Alexandre

1894.Hygiène industrielle, in Encyclopédie d'Hygiène et de médecine publique, pp. 464-473, in Jules Rochard (ed.), Livre VI L. Paris : Bataille et Cie

Lefebvre, Théodore et Cie

1865.Fabrique de céruse de Théodore Lefebvre \& Cie à Lille, section des Moulins (Nord). Rapports, Notices, Documents, Extraits divers, etc. 1825-1865. Lille :Horemans.

Lefebvre, Théodore

1878. Notice sur la fabrique de céruse de MM. Théodore Lefebvre et cie à Lille (Nord). Exposition Universelle de 1878, Groupe V - classe 47 - n 4. Lille : L. Danel.

Loir, A., E. Ferrand et Radisson

1883.Rapport sur l'usine de céruse de MM. Richard et Régnier à Lyon. 
Millot-Cousin, Alfred

1878.Notice sur la Fabrique de Céruse de M. Millot-Cousin, à Lille. Exposition Universelle. Lille :

Imprimerie de Six-Horemans.

Napias, Henri

1882.L'étude et les progrès de l'hygiène en France de 1878 à 1882. Paris : Masson.

Tardieu, Ambroise

1862.Dictionnaire d'hygiène publique et de salubrité. Paris : J. B. Baillère et fils. Tome 3 (2ème édition).

Troost, Louis

1894.Précis de chimie. Paris : Masson (26ème édition).

Turgan, Julien

1885.« Usine Perus \& Cie à Lille (Nord) », Les grandes usines, tome XVI.

Viet, Vincent

1994.Les Voltigeurs de la République, l'inspection du travail en France jusqu'en 1914. Paris : Éditions du CNRS.

\section{NOTES}

1. Séance du Conseil de Salubrité (Seine) du 12 novembre 1824, AN F ${ }^{12} 2428$.

2. Livre d'Honneur de l'Exposition des produits de l'industrie française en 1839, Ducessois, Paris (1840), cité dans Fabrique de céruse de Théodore Lefebvre \& Cie à Lille, section des Moulins (Nord), Rapports, Notices, Documents, Extraits divers, etc. 1825-1865, Horemans, Lille (1865), p.51.

3. Cf. dossier Céruse $\mathrm{AN} \mathrm{F}^{12} 2424$ : la fabrication de plomb « à la manière hollandaise » est avérée chez Migneron de Brocqueville, à Bordeaux puis à Paris, 25 rue d'Enfer, en 1790, et dans la Manufacture de blanc de céruse de Simon Léon Cauzauranc, à Lagnysur-Marne et rue de Vaugirard, Paris, en 1791. En 1827, Théodore Lefebvre accrédite M. Chaillot, de Paris, de l'importation du procédé de fabrication de la céruse à la manière hollandaise en 1791, date « qui fut pour ainsi dire le signal de l'érection de nombreuses fabriques ». (Cf. Lefebvre et Cie $1865: 33$.)

4. Archives Départementales (AD) 59 : dossiers des établissements classés sous série M417; Almanach du Commerce du Département du Nord 1854-1970.

5. Archives Nationales (AN) FA2 7609 et 7610.

6. Lettre du 22 mai 1898 au Préfet du Nord, AD59 M417-5089.

7. Trois références à Théodore Lefebvre sont citées : la première est anonyme et sera référencée sous le nom Lefebvre et Cie; les deux autres sont le fait du successeur du fondateur de la fabrique, un parent du même nom, qui prit la suite en 1864.

8. $\mathrm{AN} \mathrm{F}^{12} 6853$ et Rapport du Jury mixte international, Exposition Universelle de 1855 (cité par Lefebvre et Cie $1865: 17$ ).

9. Meurein, Rapport du 12 juillet 1876 (Lefebvre 1878 : 10) ; Gautier (1883 : 251) ; Jules Arnould, in Congrès International d'hygiène tenu à Paris $d u 1^{\text {er }}$ au 10 août 1878, Imprimerie Nationale, paris (1880 tome $1: 638$ ).

10. Le pionnier est la fabrique de Théodore Lefebvre, qui s'est attaché les services du Dr Degland, autorisé à faire délivrer, aux frais de l'établissement, tous les médicaments 
jugés convenables au rétablissement des ouvriers dès le moindre signe de maladie. ( $C f$. Lefebvre et Cie 1865 : 38.)

11. Exposition des produits de l'industrie française en 1844, Rapport du jury central, Paris 1844 , 3 vol. (vol $2: 734)$.

12. Académie Nationale, séance du 5 janvier 1873 (cité par Lefebvre $1878: 6$ ).

13. AN $\mathrm{F}^{12}$ : Administration générale des Hospices et Hôpitaux civils de Paris, « Etat des Peintres et des ouvriers employés à la fabrication de la céruse atteints de la colique de plomb, admis pendant les années 1818, 1819, 1820, 1821, 1822 dans les hôpitaux dépendant de la $1^{\text {ère }}$ et de la $2^{\text {ème }}$ division « (3 tableaux) : Chevallier 1849:6); Tardieu (1862 : 345) ; Rapport général sur les travaux du Conseil d'hygiène du Département de la Seine, 1867-1871 : 23; Gautier $1883: 237-252$ ).

14. Un pourcentage supérieur à 100 signifie que les fabriques envoient chaque année à l'hôpital plus de malades qu'elles n'emploient d'ouvriers, soit qu'un ouvrier y retourne plusieurs fois, soit que le turn-over des ouvriers est grand.

15. Ce que Gautier (1883:247) interprète par « chaque ouvrier de cette fabrique passe en moyenne quatre fois par an à l'hôpital ! »

16. Article communiqué par G. Jorland, que je remercie.

17. De 1826 à 1842, le nombre de malades chez Théodore Lefebvre est de 30 à 35 par an pour 100 à 110 ouvriers employés, d'après une attestation du Dr Degland, médecin attaché à la fabrique (cité par Lefebvre et Cie 1865 : 95).

18. Bulletin de la Société pour l'Encouragement de l'Industrie Nationale, 51 :129, 539 (1852); Lefebvre et Cie (1865: 195).

19. Exposition des produits de l'industrie française en 1844, Rapport du jury central (Paris 1844; 3 vol., vol. $2: 749$ ).

20. Arrêté préfectoral du 10 juin 1898.

21. AD 59 M417-5089.

22. Elles sont décrites sur la planche XXIV concernant la fabrication de la céruse $d u$ Précis de Chimie industrielle de Payen en 1851. Je remercie G. Emptoz de m'avoir signalé cette information; AD59 M417.

23. Exposition des produits de l'industrie française en 1844, Rapport du jury central, Paris (1844, 3 vol., vol. 2 : 739, 749).

24. Rapport général sur les travaux du Conseil d'hygiène (1867-1871 : 26) ; AD59 M417 5089).

25. Théodore Lefebvre et Cie, Brevet d'invention de quinze ans n 8374, délivré le 27 juillet 1849, sur dépôt en date du 11 mai 1849 « pour un nouvel appareil à pulvériser la céruse ».

26. Lettre du 15 décembre 1823 de Mouvet et Mathieu, fabricants de céruse, au ministre de l'Intérieur, AN F12 2428.

27. AD59 M417 5140.

28. Procès Verbal de récolement, 11 février 1893, AD59 M417 5140 et 14 janvier 1899, AD59 M417 5089.

29. AD59 10R 5547; AD59 M417 4449 et Freycinet (1870 : 73).

30. AD59 M417 5089.

31. Exposition des produits de l'industrie française en 1844, Rapport du jury central, Paris 1844, 3 vol. (vol. 2 : 729).

32. Rapport général sur les travaux du Conseil d'hygiène publique et de salubrité, 1872-1877, p. 835. 
33. 1834 : Première instruction du Conseil de Salubrité de la Seine concernant la fabrication de la céruse, PV des travaux du Conseil de salubrité, 1829-1839, pp. 70-71 (communication d'Anne-Cécile Lefort); Instructions sur les précautions à mettre en usage dans les fabriques de blanc de plomb, pour y rendre le travail moins insalubre, 14 avril 1837, Archives de Paris DM5.

34. Instructions du Conseil d'Hygiène de la Seine concernant la fabrication et la manipulation du plomb et de ses composés, 23 décembre 1881, édictées en circulaire du préfet en date du 24 janvier 1882 (cité par Napias 1882 : 103).

35. Décret du 18 juillet 1902, réglementant l'emploi du blanc de céruse dans l'industrie de la peinture en bâtiment.

36. L'usage de bois ayant été enduits de peinture ou ayant subi des préparations chimiques quelconques est interdit à Paris pour le chauffage des fours de boulangerie et de pâtisserie, depuis l'ordonnance de police du 15 septembre 1877, consécutive à une « épidémie » d'intoxication saturnine dans le XVIIe arrondissement, qui a touché la clientèle d'un boulanger qui chauffait son four avec des bois de démolition.

37. AD93 219W6; AD59 M417 16224.

38. Clovis Hugues (1851-1907); poème écrit alors qu'il est député de Paris et membre de la Commission Parlementaire sur la céruse, et publié à titre posthume.

\section{RÉSUMÉS}

La céruse est le pigment blanc le plus commun du XIXe siècle. C'est un carbonate de plomb, produit en France de manière industrielle depuis 1809, date de la fondation de l'usine de Clichy. C'est cependant la région lilloise qui prédomine dans ce secteur où seul le procédé traditionnel « hollandais » est employé. Conscients de la dangerosité de ce métier, qui provoque le saturnisme de nombreux ouvriers, les cérusiers, menés par Théodore Lefebvre, œuvrent avec succès pour rendre leur industrie plus saine, en introduisant de nombreuses innovations destinées à réduire la poussière émise. Ils répondent ainsi aux sollicitations des hygiénistes et de l'État, qui finira par interdire l'utilisation de la céruse par les peintres en bâtiment au début du XXe siècle. Clichy, qui s'est fait une spécialité de la céruse en poudre, demeure tout au long du XIXe siècle l'usine la plus insalubre jusqu'à sa fermeture en 1882 .

The production of white-lead in France in the 19th century: evolution of a dangerous industry

Ceruse was the most common white pigment in the 19th century. It is a lead carbonate which was industrially produced in France since 1809 when first factory opened in Clichy in the suburbs of Paris. Its fabrication was however a speciality of the Lille region in the North of France. There, it was made exclusively according to the traditionnal « Dutch » process. Conscious of the risks of saturnism linked to their job, the producers, led by Théodore Lefebvre, found ways to render their activity less harmful, in particular by innovating in the field of dust reduction. By doing so, the producers responded to the appeals of the hygienists and of the State. The use of ceruse whitewashing was finally forbidden at the turn of the 20th century. Clichy, specialised in powdered white-lead, remained the most insalubrious factory up to its closing-down in 1882. 
La producción de cerusa en Francia durante el siglo XIX : evolución de una industria peligrosa

La cerusa es el pigmento blanco más común del siglo XIX. Es un carbonato de plomo, producido industrialmente en Francia desde 1809, año de la creación de la fábrica de Clichy. Sin embargo, su producción, la domina la región de Lille, donde el procedimiento " holandés » es la única técnica que se aplica. Conscientes de los peligros de la profesión, que provoca el saturnismo de gran número de obreros, los « ceruseros ", guiados por el ejemplo de Théodore Lefebvre, trabajan con éxito para sanear su industria : implantan numerosas innovaciones con objeto de reducir el polvo emitido. Así responden a las exigencias de los higienistas y del Estado que, finalmente, acaba por prohibir, a principios del siglo XX, el uso de la cerusa a los pintores de brocha gorda. Clichy, que se había especializado en la cerusa en polvo, continúa siendo la fábrica más insalubre a lo largo del siglo XIX, hasta que la cierren en 1882.

INDEX

Mots-clés : céruse, saturnisme, Lefebvre (Théodore), industrie, XIXe siècle

Keywords : ceruse, saturnism, Théodore Lefebvre, industry, 19th century

\section{AUTEUR}

\section{LAURENCE LESTEL}

Centre d'Histoire des Techniques, CNAM-EHESS, 5 rue du Verbois, 75003Paris 